\title{
ON THE INTERPRETATION OF THE NEEDLE PROBE TEST RESULTS: THERMAL CONDUCTIVITY MEASUREMENT OF CLAYEY SOILS
}

\author{
ADRIAN RÓŻAŃSKI, MACIEJ SOBÓTKA \\ Institute of Geotechnics and Hydrotechnics, Wrocław University of Technology, \\ Wybrzeże Wyspiańskiego 27, 50-370 Wrocław, Poland. \\ E-mail: adrian.rozanski@pwr.wroc.pl,maciej.sobotka@pwr.wroc.pl
}

\begin{abstract}
Recently, there is an increasing need for accurate and rapid thermal measurement of soils. Within a variety of available methods a needle probe test is most widely used. The needle probe method was standardized for the measurement of thermal conductivity of soils and soft rocks. In the paper, two different interpretation methods of the needle probe test were used for determination of thermal conductivity of selected soils. The first method (ASTM D5334-05 Standard approach) takes into account only the data which are recorded during heating while the second approach is based on fitting the known analytical solution to the data obtained within both heating and cooling phases. The soil samples used were classified as clayey ones. Laboratory tests were performed using the KD2Pro thermal conductivity meter (Decagon Devices) with a TR-1 sensor. The main goal of the paper is to show that the selection of interpretation method is very important and may lead to significant differences in resulting thermal conductivities.
\end{abstract}

\section{INTRODUCTION}

Soil or rock thermal properties are recently of great importance in many engineering projects, e.g., in design of geothermal heating and cooling systems, pipelines, buildings in cold regions, underground power cables or coal gasification, etc. [6]. In general, these properties are important in every situation where heat transfer takes place in the soils or rocks.

Both thermal conductivity and specific heat are the main parameters affecting the transfer of heat energy through a given medium. This heat transfer is commonly described as the conductive flow. However, in many cases such mechanisms as convection or radiation also contribute to the overall transfer - this is particularly common in fluids or gases. For solids or particulates, the conductive mechanism overwhelmingly controls. It should also be mentioned that such phenomena as water phase changes and their associated energy very often have a significant effect on the process of heat transfer in soils [6]. In addition, as the most difficult problem in the field of soil heat transfer, De Vries [4] has pointed out the case of the combined (simultaneous) transfer of heat and moisture.

Due to the aforementioned interest in soil thermal properties there is an increasing need for accurate and rapid thermal measurement of soils. Generally, the methods for measuring thermal conductivity can be classified into two categories, i.e., 
steady-state and transient heat transfer methods [11]. Steady-state methods often require a long time to complete as a sample or soil portion being tested should be in a steady state when the measurements are made. On the contrary, transient methods usually require much less time and, in addition, are more versatile and easily performed [5]. Recently, the line source method is most widely used. Within this method a steel needle probe, as a heating source, inserted into soil material is usually utilized. During the measurement the probe is heated and the change in temperature, for heating and cooling phases, is recorded. In general, the probe mimics a transient-line heat source for which an analytical solution has been obtained by Carslaw and Jaeger [3]. A large number of scientists have used the needle probe for determining thermal conductivity of soils (e.g., [12], [8]), snow (e.g., [13]) or materials from food industry (e.g., [7]).

The needle probe method was standardized for soils and soft rocks ([2], [9]). Here the focus is on the influence of the interpretation method on the resulting thermal conductivity for samples of clayey soils. Both aforementioned standards, namely [2] and [9], propose determining thermal conductivity on the basis of the temperatures recorded only for the heating cycle. Roughly speaking, this type of interpretation consists in plotting the data on a semi-log graph, selecting a portion of the data by eye that appears to fit a straight line, selecting two points on that line and calculating the thermal conductivity from the slope of the straight line. It is shown in the paper that taking into account temperature rise data for both heating and cooling phases may lead to significant differences in thermal conductivities when compared to the results obtained using approaches presented in [2] and [9].

The soils used for laboratory experiments were classified as clayey ones. For each soil sample the needle probe test was performed and the results obtained for two different interpretation methods are compared. The device used for thermal conductivity measurement is the KD2Pro thermal conductivity meter with a TR-1 sensor [10]. The paper is organized as follows. Section 2 provides a brief theory background concerning the problem of temperature increase of an infinite linear constant heat source within an infinite medium. In Section 3, two different interpretation methods are presented. In Section 4, the results of the measurements as well as thermal conductivities obtained are provided. The differences between estimated conductivities are calculated and discussed. Final conclusions end the paper.

\section{THEORY OF THE PROBE METHOD}

The theory of the probe method is based on the solution of the line heat source placed within an infinite, isotropic and homogenous medium of thermal diffusivity $D$. For such medium the heat flows from the source in accordance to the general Fourier equation. For cylindrical coordinates, corresponding to the expanding radial field around the probe, the equation is 


$$
\frac{\partial T}{\partial t}=D\left(\frac{\partial^{2} T}{\partial r^{2}}+\frac{1}{r} \frac{\partial T}{\partial r}\right)
$$

where $T$ is the temperature at time $t$ and $r$ is the radial distance from the line source. Assuming that the heat is produced from time $t=0$ at a constant rate $q$ per unit length of probe, Carslaw and Jaeger [3] obtained the analytical solution, namely the temperature increase (or decrease) $\Delta T$ of the medium

$$
\begin{gathered}
\Delta T=-\frac{q}{4 \pi k} \operatorname{Ei}\left(-\frac{r^{2}}{4 D t}\right), \quad 0<t<t_{h}, \\
\Delta T=-\frac{q}{4 \pi k}\left[-\operatorname{Ei}\left(-\frac{r^{2}}{4 D t}\right)+\operatorname{Ei}\left(-\frac{r^{2}}{4 D\left(t-t_{h}\right)}\right)\right], \quad t>t_{h} .
\end{gathered}
$$

Note that equations (2) and (3) describe the temperature changes during heating and cooling, respectively. In the relations above $q$ is the heat input rate $[\mathrm{W} / \mathrm{m}], k$ represents thermal conductivity $[\mathrm{W} /(\mathrm{m} \mathrm{K})], D$ is the thermal diffusivity $\left[\mathrm{m}^{2} / \mathrm{s}\right]$ and $t_{h}$ is the heating time $[\mathrm{s}]$. Furthermore, $\operatorname{Ei}(\alpha)$ is the exponential integral

$$
-\operatorname{Ei}(-\alpha)=\int_{\alpha}^{\infty} \frac{e^{-t}}{t} d t
$$

which can be approximated by the series expansion [1]

$$
-\operatorname{Ei}(-\alpha)=-\gamma-\ln \alpha-\sum_{\alpha}^{\infty} \frac{(-\alpha)^{n}}{n n !}
$$

where $\gamma=0.5772 \ldots$ is Euler's constant and $\alpha=\frac{r^{2}}{4 D t}$.

\section{THE NEEDLE PROBE TEST INTERPRETATIONS}

As mentioned in the Introduction, both [2] and [9] standards propose to determine thermal conductivity on the basis of temperature data recorded only for the heating phase. Interpretation method is based on the fact that, for long times when $r$ is small and $D$ is large, the terms beyond $\ln \alpha$ in the series expansion of Ei (5) become negligibly small. Hence, $\Delta \mathrm{T}$ is linearly related to $\ln t$ and equation (2) can be approximated as 


$$
\Delta T \approx \frac{q}{4 \pi k} \ln t+C, \quad 0<t<t_{h},
$$

where $C$ is a constant. Therefore, performing an interpretation proposed by ASTM D5334-05 [2] one has to plot all data as a function of time on a semilog graph. Then one has to select linear portion of the curve and draw a straight line through the points. The linear portion of curve is treated as a quasi-steady state one. Next, the times $t_{1}$ and $t_{2}$ at appropriate points on the line have to be selected and corresponding temperatures $T_{1}$ and $T_{2}$ should be read. Finally, thermal conductivity $k$ is calculated using the slope $S$ of the function (6), i.e.,

$$
k=\frac{q}{4 \pi S},
$$

where

$$
S=\frac{T_{2}-T_{1}}{\ln t_{2}-\ln t_{1}}
$$

Contrary to ASTM D5334-05 approach, another proposition is to take into account both heating and cooling phases. Then the resulting data are fit to the following equations [10]

$$
T=m_{0}+m_{2} t+m_{3} \ln t
$$

for heating, and

$$
T=m_{1}+m_{2} t+m_{3} \ln \left(\frac{t}{t-t_{h}}\right)
$$

for cooling phase. In the equations above, $m_{0}$ can be treated as the ambient temperature, $m_{2}$ is the rate of background temperature drift, and $m_{3}$ is the slope of a line that relates temperature increase to $\ln t$.

Since equations (9) and (10) are long time approximations it is proposed to ignore early time data. Speaking more precisely, only the final $2 / 3$ of all the data collected (during heating and cooling) are used for fitting. While the parameters $m_{0}, m_{1}, m_{2}$ and $m_{3}$ are found (here the least squares analysis using Mathematica [14] is performed), the conductivity is calculated as:

$$
k=\frac{q}{4 \pi m_{3}} .
$$




\section{LABORATORY INVESTIGATIONS AND RESULTS}

\subsection{THERMAL CONDUCTIVITY METER}

Laboratory tests were performed using the KD2Pro thermal conductivity meter (Decagon Devices) with a TR-1 sensor (2.4 mm in diameter $\times 100 \mathrm{~mm}$ in length). The device, namely the KD2Pro meter, as well as TR-1 sensor are presented in Fig. 1. For thermal conductivity determination the range of measurement of TR-1 sensor is: 0.10 to $4.00 \mathrm{~W} /(\mathrm{mK})$, while the accuracy is: $\pm 10 \%$ from $0.2-4 \mathrm{~W} /(\mathrm{mK})$ and $\pm 0.02 \mathrm{~W} /(\mathrm{mK})$ from $0.1-0.2 \mathrm{~W} /(\mathrm{mK})[10]$.

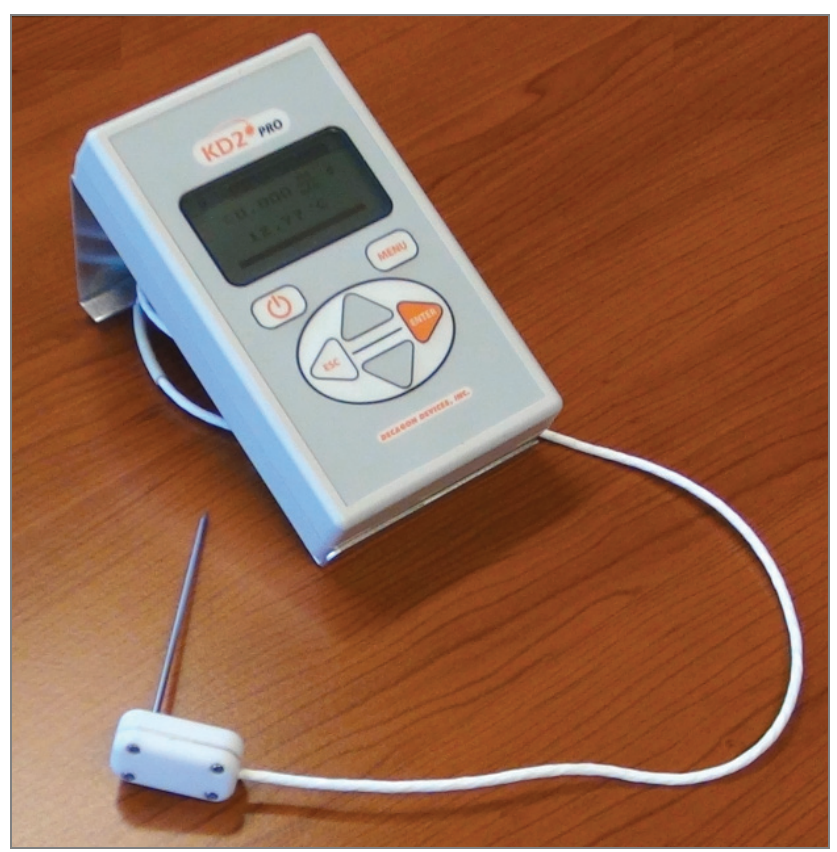

Fig. 1. KD2Pro thermal conductivity meter and TR-1 sensor

\subsection{CHARACTERIZATION OF SOILS INVESTIGATED}

Ten soil samples were selected for laboratory tests. As mentioned in the Introduction, the soils used for laboratory investigations were classified as clayey ones. Each soil sample is a cylindrical core having a radius equal to $7-7.5 \mathrm{~cm}$ and a height of 35 to $50 \mathrm{~cm}$. The sample notations as well as water content and densities are summarized in Table 1. 
Table 1

Notation, moisture content and density of clayey soils investigated

\begin{tabular}{|c|c|c|}
\hline Sample & $\begin{array}{c}\text { Moisture content } \\
w[\%]\end{array}$ & $\begin{array}{c}\text { Density } \\
\rho\left[\mathrm{g} / \mathrm{cm}^{3}\right]\end{array}$ \\
\hline C1 & 27.2 & 1.83 \\
C2 & 35.4 & 1.79 \\
C3 & 24.3 & 2.08 \\
C4 & 28.5 & 1.97 \\
C5 & 52.5 & 1.93 \\
C6 & 21.9 & 2.14 \\
C7 & 17.2 & 2.03 \\
C8 & 21.6 & 2.01 \\
C9 & 24.5 & 1.98 \\
C10 & 20.0 & 2.10 \\
\hline
\end{tabular}

\subsection{RESULTS}

For each soil sample the needle probe test was conducted and the interpretation of obtained data, using both aforementioned approaches (ASTM D5334-05 Standard proposition - only heating phase vs. both heating and cooling phase fitting), was performed. The heating time, $t_{h}$, is set to 150 seconds. In Figs. $2-11$, the resulting data for all 10 tests are graphically presented. In each figure, on the left, only a linear portion of heating curve for ASTM D5334-05 Standard interpretation is shown. On the right, all recorded data (for both heating and cooling phases) as well as fitting functions (9)-(10) are provided. Since only the final $2 / 3$ of collected data are used for fitting the first $1 / 3$ are presented by grey (instead of black) dots.
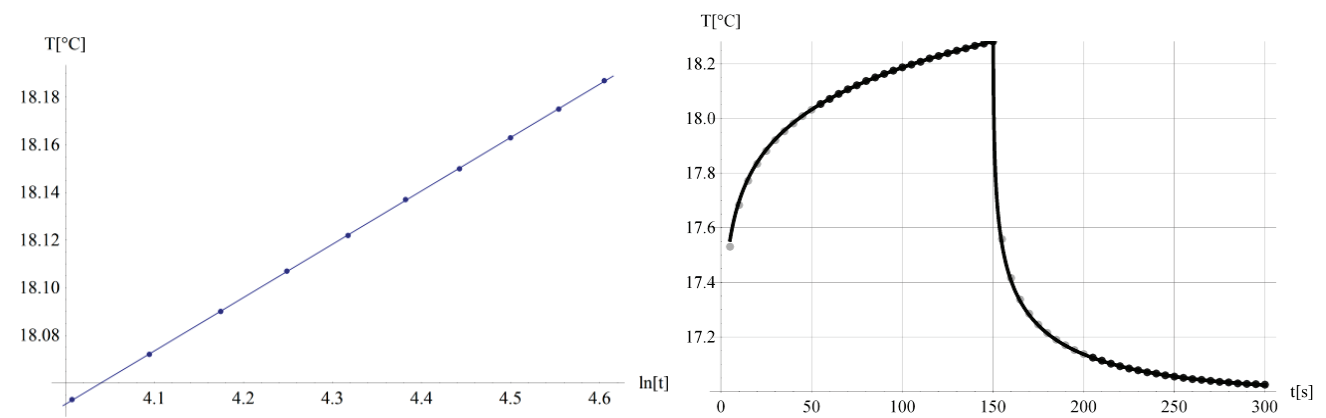

Fig. 2. Resulting data (temperature vs. time) for sample C1.

Left: linear portion of curve for ASTM D5334-05 Standard interpretation.

Right: fitting function for heating and cooling phase 

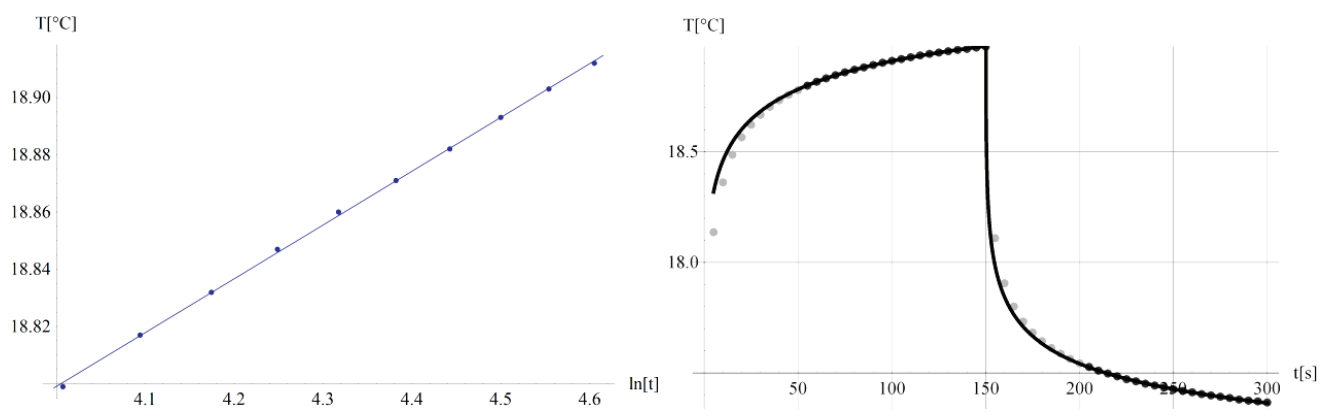

Fig. 3. Resulting data (temperature vs. time) for sample $\mathrm{C} 2$.

Left: linear portion of curve for ASTM D5334-05 Standard interpretation.

Right: fitting function for heating and cooling phase
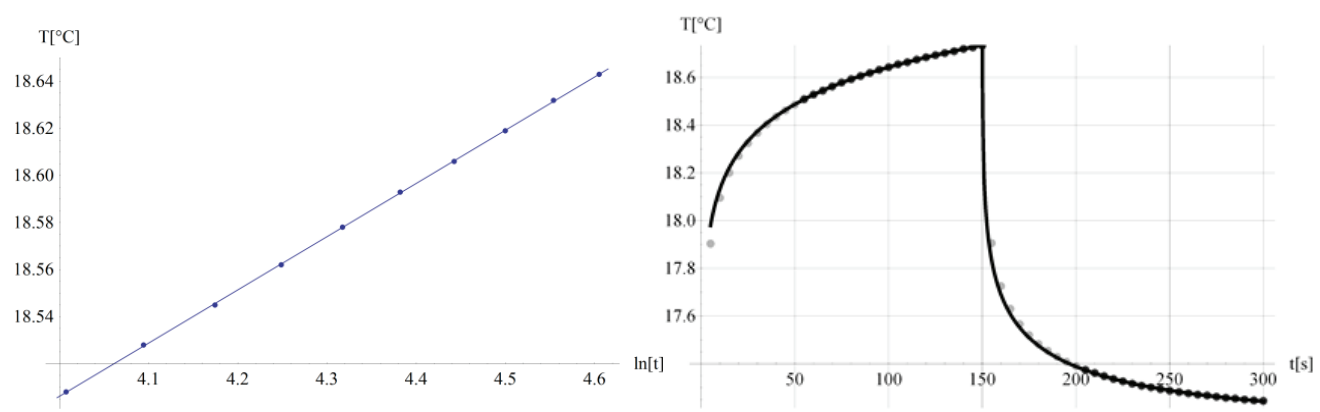

Fig. 4. Resulting data (temperature vs. time) for sample C3.

Left: linear portion of curve for ASTM D5334-05 Standard interpretation.

Right: fitting function for heating and cooling phase

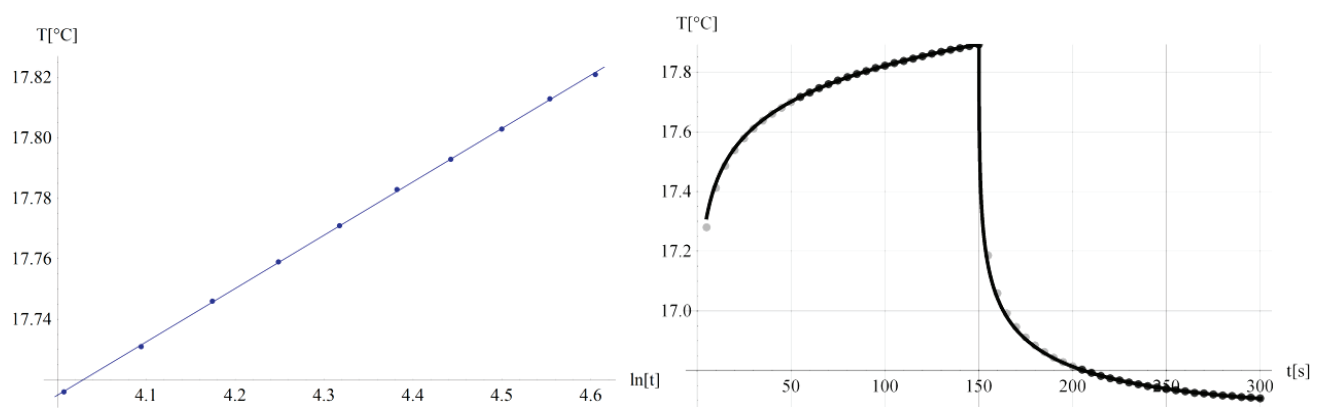

Fig. 5. Resulting data (temperature vs. time) for sample C4.

Left: linear portion of curve for ASTM D5334-05 Standard interpretation.

Right: fitting function for heating and cooling phase 

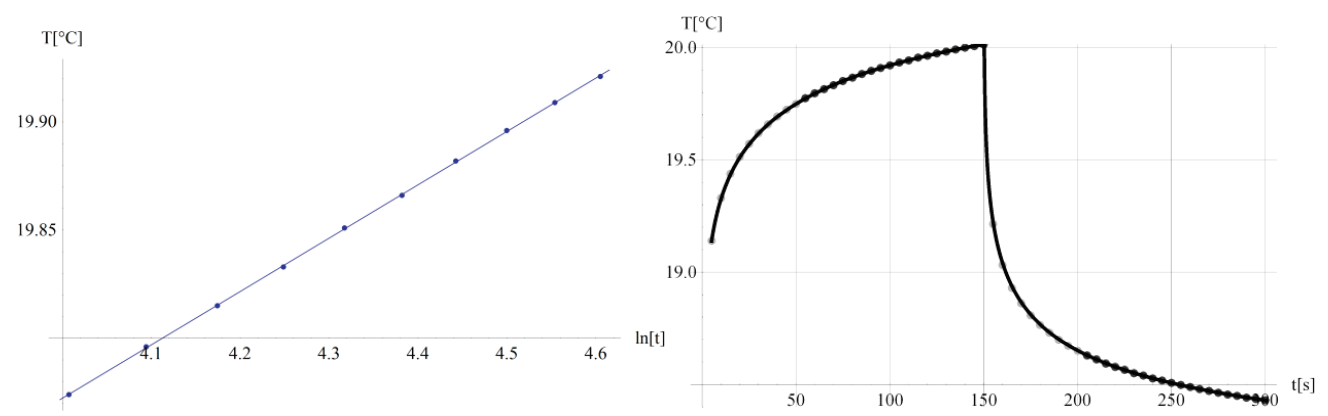

Fig. 6. Resulting data (temperature vs. time) for sample C5.

Left: linear portion of curve for ASTM D5334-05 Standard interpretation.

Right: fitting function for heating and cooling phase

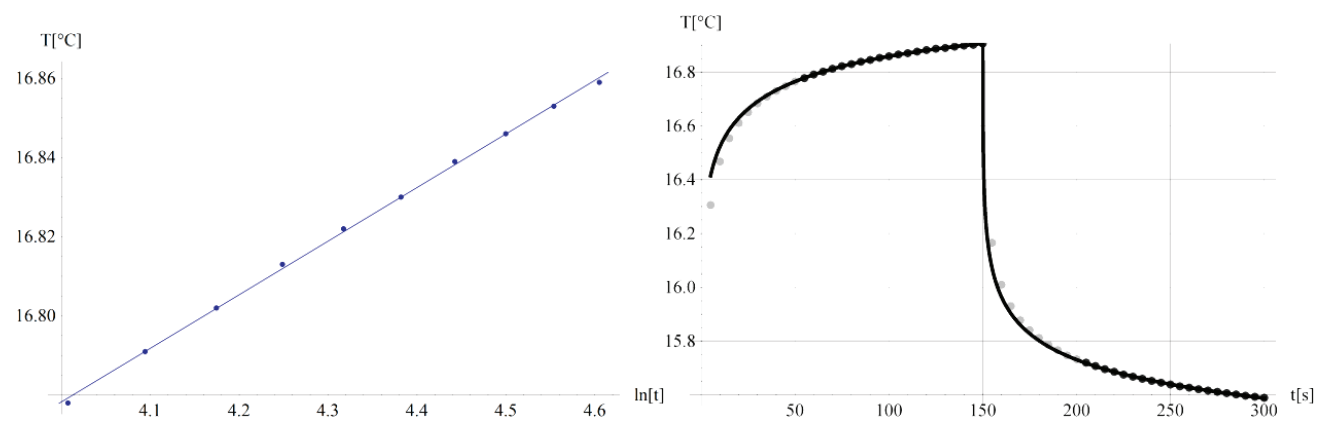

Fig. 7. Resulting data (temperature vs. time) for sample C6.

Left: linear portion of curve for ASTM D5334-05 Standard interpretation.

Right: fitting function for heating and cooling phase
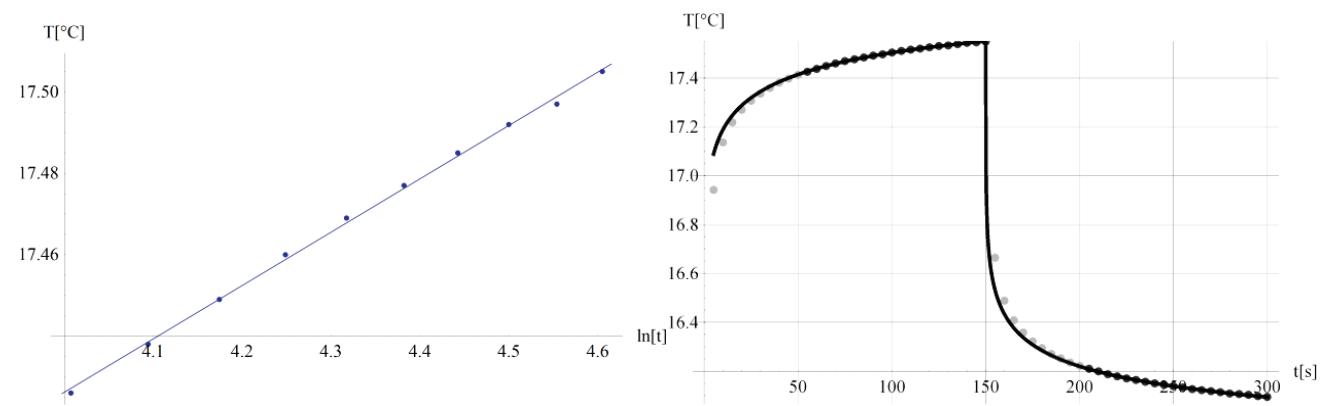

Fig. 8. Resulting data (temperature vs. time) for sample C7.

Left: linear portion of curve for ASTM D5334-05 Standard interpretation.

Right: fitting function for heating and cooling phase 


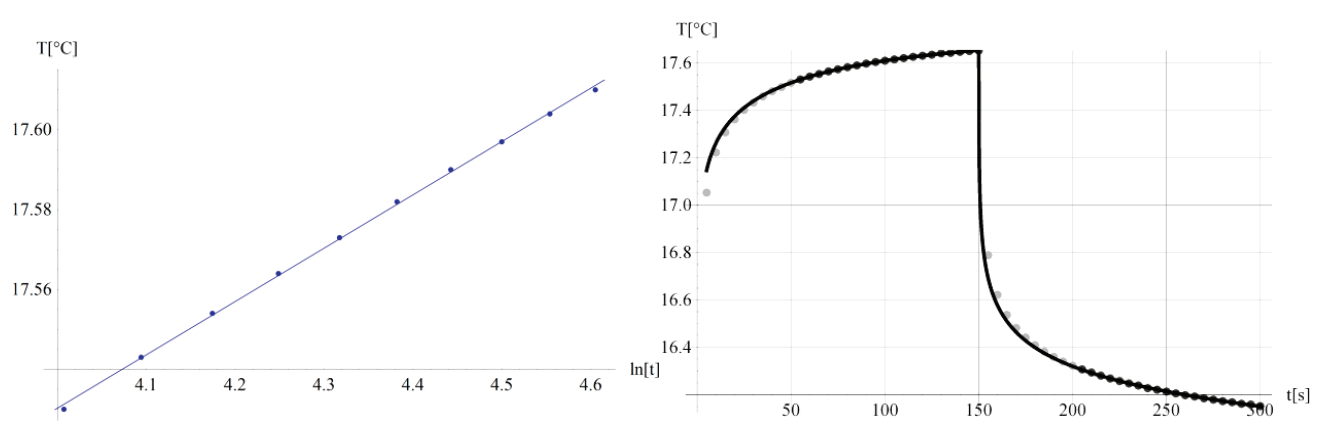

Fig. 9. Resulting data (temperature vs. time) for sample C8.

Left: linear portion of curve for ASTM D5334-05 Standard interpretation.

Right: fitting function for heating and cooling phase

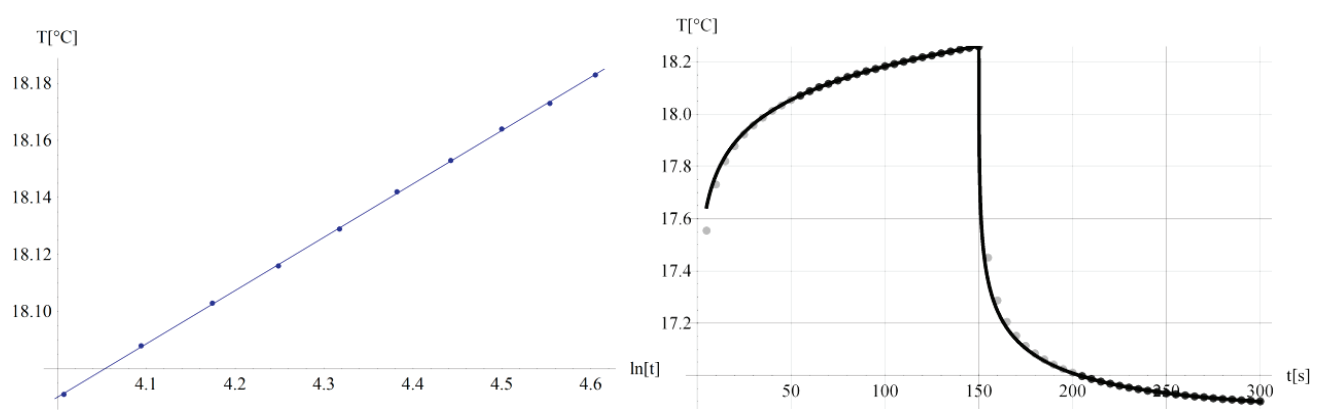

Fig. 10. Resulting data (temperature vs. time) for sample C9.

Left: linear portion of curve for ASTM D5334-05 Standard interpretation.

Right: fitting function for heating and cooling phase

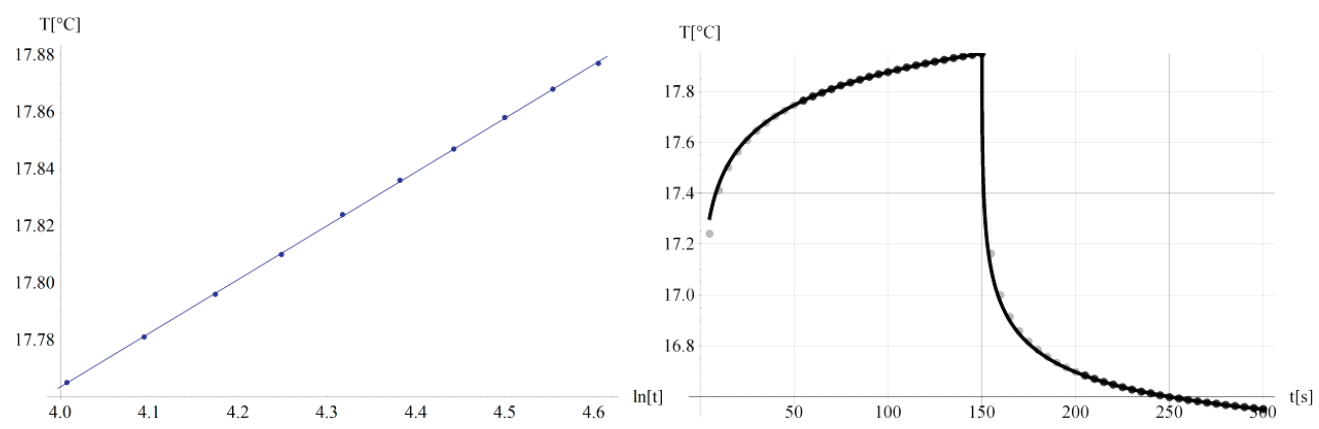

Fig. 11. Resulting data (temperature vs. time) for sample C10. Left: linear portion of curve for ASTM D5334-05 Standard interpretation. Right: fitting function for heating and cooling phase

Table 2 summarizes interpretation data as well as the values of thermal conductivities $\left(k_{A S T M}\right)$ obtained in accordance with ASTM D5334-05 Standard - relation (7). In Table 2, such data as the slope of the straight line $S$ (determined using equation (8)) 
and the heat input rate $q$ are provided. Thermal conductivity ranged from 1.245 $[\mathrm{W} /(\mathrm{mK})]$ for sample $\mathrm{C} 5$ to $2.445[\mathrm{~W} /(\mathrm{mK})]$ for sample C7. Note that C5 and C7 are the soil samples having the greatest and the lowest moisture content (see Table 1), respectively.

Table 2

Slope, heat input rate and calculated thermal conductivity in the case of heating curve fitting (ASTM D5334-05 Standard approach)

\begin{tabular}{|c|c|c|c|}
\hline Sample & $\begin{array}{c}\text { Slope } \\
\text { of the straight line } S\end{array}$ & $\begin{array}{c}\text { Heat input } \\
\text { rate } q[\mathrm{~W} / \mathrm{m}]\end{array}$ & $\begin{array}{c}\text { Thermal conductivity } \\
k_{A S T M}[\mathrm{~W} /(\mathrm{mK})]\end{array}$ \\
\hline $\mathrm{C} 1$ & 0.224 & 3.940 & 1.399 \\
$\mathrm{C} 2$ & 0.188 & 3.870 & 1.639 \\
$\mathrm{C} 3$ & 0.226 & 3.930 & 1.382 \\
$\mathrm{C} 4$ & 0.177 & 4.020 & 1.812 \\
$\mathrm{C} 5$ & 0.247 & 3.860 & 1.245 \\
$\mathrm{C} 6$ & 0.135 & 3.580 & 2.104 \\
$\mathrm{C} 7$ & 0.131 & 4.030 & 2.445 \\
$\mathrm{C} 8$ & 0.134 & 4.100 & 2.443 \\
$\mathrm{C} 9$ & 0.187 & 4.080 & 1.737 \\
$\mathrm{C} 10$ & 0.189 & 3.890 & 1.641 \\
\hline
\end{tabular}

In Table 3, the data for interpretation method which takes into account both heating and cooling phases, are shown. Table 3 presents, in addition, the values of thermal conductivities $k$ calculated using equation (11). Note that the heat input rate is the same as presented in Table 2. Again, the minimum and maximum values of thermal conductivity were obtained for samples $\mathrm{C} 5$ and $\mathrm{C} 7$, respectively; thermal conductivity ranged from 1.131 to $2.190[\mathrm{~W} /(\mathrm{mK})]$.

Table 3

Fitting parameters $m_{0}, m_{1}, m_{2}, m_{3}$ and calculated thermal conductivity $k$ in the case of heating and cooling curve fitting

\begin{tabular}{|c|c|c|c|c|c|}
\hline \multirow[t]{2}{*}{ Sample } & \multicolumn{4}{|c|}{ Fitting parameters } & \multirow{2}{*}{$\begin{array}{c}\text { Thermal conductivity } \\
k[\mathrm{~W} /(\mathrm{mK})]\end{array}$} \\
\hline & $m_{0}$ & $m_{1}$ & $m_{2}$ & $m_{3}$ & \\
\hline $\mathrm{C} 1$ & 17.2286 & 16.8012 & 0.000275606 & 0.202085 & 1.552 \\
\hline $\mathrm{C} 2$ & 17.9850 & 17.3191 & -0.00033223 & 0.208234 & 1.479 \\
\hline $\mathrm{C} 3$ & 17.6261 & 17.0711 & 0.000065287 & 0.219310 & 1.426 \\
\hline $\mathrm{C} 4$ & 17.0431 & 16.5649 & 0.000087288 & 0.166999 & 1.916 \\
\hline $\mathrm{C} 5$ & 18.7029 & 18.3411 & -0.00033161 & 0.271698 & 1.131 \\
\hline C6 & 16.1609 & 15.5761 & -0.00032835 & 0.158750 & 1.795 \\
\hline $\mathrm{C} 7$ & 16.8544 & 16.0661 & -0.00024615 & 0.146421 & 2.190 \\
\hline $\mathrm{C} 8$ & 16.8740 & 16.1838 & -0.00050567 & 0.170741 & 1.911 \\
\hline C9 & 17.3620 & 16.7393 & 0.000130388 & 0.175429 & 1.851 \\
\hline $\mathrm{C} 10$ & 16.9886 & 16.4469 & -0.00010592 & 0.195202 & 1.586 \\
\hline
\end{tabular}


Next the relative differences between the conductivities obtained from two interpretation methods were calculated. Table 4 presents the percentage values of differences calculated as

$$
\varepsilon=\frac{\left|k-k_{A S T M}\right|}{k} \cdot 100[\%]
$$

Table 4

Relative differences between the conductivities obtained from two interpretation methods

\begin{tabular}{|c|c|}
\hline Sample & $\begin{array}{c}\text { Relative difference } \\
\varepsilon[\%]\end{array}$ \\
\hline C1 & 9.80 \\
C2 & 10.80 \\
C3 & 3.06 \\
C4 & 5.40 \\
C5 & 10.16 \\
C6 & 17.22 \\
C7 & 11.64 \\
C8 & 27.84 \\
C9 & 6.17 \\
C10 & 3.48 \\
\hline
\end{tabular}

Observing the results (Table 4) it can be seen that, in some cases, the two interpretation methods considered may lead to significant differences in resulting conductivities. While the results for samples C3, C4, C9 and C10 are comparable (relative difference ranged from $3.06 \%$ to $6.17 \%$ ), the differences for conductivites of remaining samples take rather significant values. The percentage difference $\varepsilon$ ranged from $9.80 \%$ for sample C1, up to $27.84 \%$ for sample C8. Especially, the conductivity difference between two interpretation methods is noticeable for sample C8 - ASTM D5334-05 Standard approach gives the conductivity $k_{A S T M}=2.443[\mathrm{~W} /(\mathrm{mK})]$ whereas for interpretation based on both heating and cooling phases the conductivity is $k=1.911[\mathrm{~W} /(\mathrm{mK})]$.

\section{CONCLUSIONS}

In the paper, two different interpretation methods of the needle probe test were used for determination of thermal conductivity of clayey soils. The results obtained were compared. The first method considered (ASTM D5334-05 Standard approach) takes into account only the data which are recorded within the heating phase. In other words, the temperature vs. time plot during cooling phase is ignored. The second approach is based 
on fitting the known analytical solution ([3], [10]) to the data obtained within both heating and cooling periods.

Ten samples of clayey soils were selected for laboratory tests. In the case of four soils investigated the resulting conductivities are comparable - relative differences between the two interpretation methods ranged from $3.06 \%$ to $6.17 \%$. The next four soils exhibit the difference at the level of $10-11 \%$. What is most important, in two cases, i.e., for the samples denoted as C6 and C8, the relative differences are $17.22 \%$ and $27.84 \%$, respectively. Furthermore, we cannot clearly state that one method overestimates/underestimates the other one.

The main goal of the paper was not to decide on the superiority of one method over the other. However, the results presented in the paper showed that the selection of interpretation method (for the needle test probe) is very important and may lead to significant differences in resulting thermal conductivites. The ASTM D5334-05 Standard approach is certainly much easier to use, as the fitting of heating phase results is performed by a straight line, but on the other hand, selecting a portion of the data by eye that appears to fit a straight line is rather moot. Whithin the second approach both heating and cooling data have to be recorded. Moreover, fitting is more difficult, as four parameters have to be determined for functions (9) and (10). However, it seems that taking into account both phases of measurement may lead to a "smoother" result when compared to the approach proposed by ASTM D5334-05 Standard.

\section{REFERENCES}

[1] Abramowitz M., Stegun I.A., Handbook of mathematical functions, Dover Publications, Inc., New York 1972.

[2] ASTM D5334-05 Standard, Standard Test Method for Determination of Thermal Conductivity of Soil and Soft Rock by Thermal Needle Probe Procedure, 2005.

[3] Carslaw H.S., Jaeger J.C., Conduction of heat in solids, Second edition, Oxford, Clarendon Press, 1959.

[4] De VRIES D.A., Heat transfer in soils, [in:] Heat and Mass Transfer in the Biosphere, I. Transfer Processes in Plant Environment, John Wiley \& Sons, New York 1974.

[5] De VRies D.A., Peck A.J., On the cylindrical probe method of measuring thermal conductivity with special reference to soils, Austr. Journal of Physics, Vol. 11, 1958.

[6] Farouki O.T., Thermal Properties of Soils, CRREL Monograph 81-1, New Hampshire 1981.

[7] Fontana A.J., Varith J., Ikediala J., Reyes J., Wacker B., Thermal properties of selected foods using a dual needle heat-pulse sensor, written for Presentation at the 1999 ASAE/CSAESCGR Annual International Meeting, Toronto, Ontario Canada, July 18-21, 1999.

[8] Hanson J.L., Edil T.B., Yesiller N., Thermal Properties of High Water Content Materials, Geotechnics of High Water Content Materials, ASTM D5334-05 STP 1374, T.B. Edil and P.J. Fox, (eds.), ASTM D5334-05 International, West Conshohocken, PA, 2000, 137-151.

[9] IEEE Guide for Soil Thermal Resistivity Measurements, 1981.

[10] KD2 Pro Thermal Properties Analyzer Operator's Manual, Version 10, Decagon Devices, Inc., 2011. 
[11] Mohsenin N.N., Thermal Properties of Foods and Agricultural Materials, Gordon and Breach, New York 1980.

[12] Nicolas J., AndR'e P., Rivez J.F., Debbaut V., Thermal Conductivity Measurements in Soil Using an Instrument Based on the Cylindrical Probe Method, Review of Scientific Instruments, Vol. 64, No. 3, 1993, 774-780.

[13] Riche F., SchneEbeli M., Microstructural change around a needle probe to measure thermal conductivity of snow, Journal of Glaciology, Vol. 56, No. 199, 2010.

[14] Wolfram Mathematica 7, Wolfram Research, Inc., 2009. 